\title{
A Log-Linearized Arterial Viscoelastic Model for Evaluation of the Carotid Artery
}

\author{
Harutoyo Hirano $^{1,2}$, Student Member, IEEE, Tetsuya Horiuchi ${ }^{3}$, Abdugheni Kutluk ${ }^{4}$, \\ Yuichi Kurita ${ }^{1}$, Member, IEEE, Teiji Ukawa ${ }^{5}$, Ryuji Nakamura ${ }^{6}$, Noboru Saeki ${ }^{6}$, Yukihito Higashi ${ }^{7}$, \\ Masashi Kawamoto ${ }^{6}$, Masao Yoshizumi ${ }^{6}$, and Toshio Tsuji ${ }^{1}$, Member, IEEE
}

\begin{abstract}
This paper proposes a method for qualitatively estimating the mechanical properties of arterial walls on a beatto-beat basis through noninvasive measurement of continuous arterial pressure and arterial diameter using an ultrasonic device. First, in order to describe the nonlinear relationships linking arterial pressure waveforms and arterial diameter waveforms as well as the viscoelastic characteristics of arteries, we developed a second-order nonlinear model (called the loglinearized arterial viscoelastic model) to allow estimation of arterial wall viscoelasticity. Next, to verify the validity of the proposed method, the viscoelastic indices of the carotid artery were estimated. The results showed that the proposed model can be used to accurately approximate the mechanical properties of arterial walls. It was therefore deemed suitable for qualitative evaluation of arterial viscoelastic properties based on noninvasive measurement of arterial pressure and arterial diameter.
\end{abstract}

\section{INTRODUCTION}

Rate of mortality from cardiovascular and cerebrovascular disease have seen an increase in recent years [1], and various noninvasive techniques for evaluating arteriosclerosis (such as pulse wave velocity (PWV) [2] and the augmentation index (AI) [3]) have been actively developed by researchers in the past. Although these indices are well known and widely used to support the evaluation of arteriosclerosis and vascular aging, their values are strongly dependent on blood pressure, heart rate, height and other factors [4], [5]. It is necessary to consider the relationship between the force acting on an artery and movement of arterial wall to verify the mechanical characteristics of an arterial wall.

Previous studies on arterial mechanical properties have indicated nonlinearity between the diameter and intravascular pressure of human arteries. Hayashi et al. proposed the

${ }^{1}$ H. Hirano, Y. Kurita, and T. Tsuji are with Division of Electrical, Systems and Mathematical Engineering, Institute of Engineering, Hiroshima University, Higashi-Hiroshima, Hiroshima, Japan. email: harutoyo@bsys.hiroshima-u.ac.jp

${ }^{2} \mathrm{H}$. Hirano is a Research Fellow of Japan Society for the Promotion of Science.

${ }^{3} \mathrm{~T}$. Horiuchi is with Department of System Cybernetics, Graduate School of Engineering, Hiroshima University, Higashi-Hiroshima, Hiroshima, Japan.

${ }^{4} \mathrm{~A}$. Kutluk is with The College of Medical Engineering Technology, Xinjiang Medical University, Urumqi, Xinjiang, China.

${ }^{5}$ T. Ukawa is with Nihon Kohden Corporation, Shinju-ku, Tokyo, Japan.

${ }^{6}$ R. Nakamura, N. Saeki, M. Kawamoto, and M. Yoshizumi are with Institute of Biomedical \& Health Sciences at Hiroshima University, Hiroshima, Japan.

${ }^{7}$ Y. Higashi is with Research Institute for Radiation Biology and Medicine, Hiroshima University, Hiroshima, Hiroshima, Japan. stiffness parameter as an intravascular pressure-independent elastic modulus [6]. However, arteries are known to have viscoelastic properties rather than elastic properties. Accordingly, many research groups have reported that the trajectory of blood pressure and arterial diameter seen during the diastolic phase is different from that observed during the systolic phase, so a Lissajous curve can be drawn for a single beat [7]. It is widely considered that arterial viscosity can be expressed by the area of a Lissajous curve, but its characteristics cannot be evaluated using only elastic parameters such as the stiffness parameter $\beta$. Barra et al. proposed a method for determining this stiffness parameter involving the removal of components depending on arterial diameter velocity and acceleration from measured arterial blood pressure values [8]. However, the relationship between arteriosclerosis and viscosity/inertia characteristics remains unclear. In addition, as this method involves two-step parameter estimation, the optimum stiffness parameter $\beta$ cannot necessarily be obtained.

Against this background, our research group has proposed indices for arterial dynamics such as stiffness, viscosity and inertia, and has evaluated vascular conditions seen during surgery or arteriosclerosis progression using equipment such as photo electronic sensors [9]. However, this approach does not consider the nonlinearity that exists between intravascular pressure and arterial diameter. In addition, as the method represents the mechanical properties of more than one artery at the same time, it cannot be applied to single channels such as the carotid artery.

To this end, here we propose a novel log-linearized arterial viscoelastic model that offers the advantage of enabling the estimation of intravascular pressure-independent arterial viscoelastic indices. In this model, the circumferential strain waveform and logarithmic blood pressure waveform are used to estimate the viscoelastic indices. It can take nonlinearity between the arterial diameter and intravascular pressure into consideration, and can be used to estimate viscoelastic indices on a beat-to-beat basis using the linear least-squares method.

\section{LOG-LINEARIZED ARTERERIAL VISCOELASTIC INDICES}

\section{A. Mechanical model}

Arterial walls have characteristics of expansion and contraction, and their mechanical properties depend on elastic 


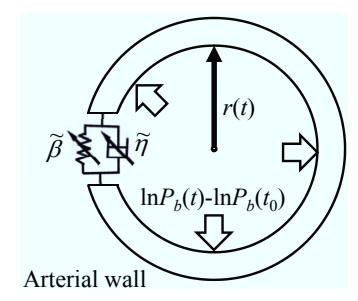

Fig. 1. Schematic model of arterial dynamics

fiber and smooth muscle fiber. In previous studies, an exponential relationship between the diameter and the intravascular pressure of human arteries was experimentally identified. The inclination of a curve corresponding to the rigidity of an arterial wall can be linearized using values of natural logarithmic blood pressure [6]. In the proposed model, the effects of blood pressure are reduced using the stiffness parameter $\beta$ as an intravascular pressure-independent elastic modulus to clarify the viscoelastic properties of arterial walls.

The stiffness parameter $\beta[6]$ is given by

$$
\beta=\frac{\ln P_{b_{s}}-\ln P_{b_{d}}}{\left(r_{s}-r_{d}\right) / r_{d}} .
$$

Here $P_{b s}, P_{b s}, r_{s}$ and $r_{d}$ are the systolic blood pressure, diastolic blood pressure, systolic arterial radius and diastolic arterial radius, respectively. This index is susceptible to disturbance from influences such as noise and body motion because it is calculated using only four values. To allow accurate evaluation of arterial wall mechanical properties, it is necessary to estimate the parameters using all these values measured continuously.

We assume that the true stiffness parameter $\tilde{\beta}$ is timeinvariant during one heartbeat and satisfies the relationship shown by Equation (1) at time $t$. This parameter is derived from the change in the blood pressure value and the arterial radius at time $t$ from initial time $t_{0}$ (for example, $\mathrm{R}$-wave timing) as follows:

$$
\tilde{\beta}=\frac{\ln P_{b}(t)-\ln P_{b}\left(t_{0}\right)}{\left\{r(t)-r\left(t_{0}\right)\right\} / r\left(t_{0}\right)} .
$$

Here $P_{b}(t)$ and $r(t)$ are the arterial pressure and radius, and $\tilde{\beta}$ is the stiffness of the arterial wall. Furthermore, $\varepsilon(t)$ is the circumferential strain, assuming that the artery is circular in its cross-sectional short axis, and is given by the following equation:

$$
\varepsilon(t)=\frac{2 \pi\left\{r(t)-r\left(t_{0}\right)\right\}}{2 \pi r\left(t_{0}\right)} .
$$

From equations (2) and (3),

$$
\ln \left(\frac{P_{b}(t)}{P_{b}\left(t_{0}\right)}\right)=\tilde{\beta} \varepsilon(t) .
$$

However, because the artery is a viscoelastic body, a viscous term is added to Equation (4) as follows:

$$
\ln \left(\frac{P_{b}(t)}{P_{b}\left(t_{0}\right)}\right)=\tilde{\beta} \varepsilon(t)+\tilde{\eta} \dot{\varepsilon}(t) .
$$

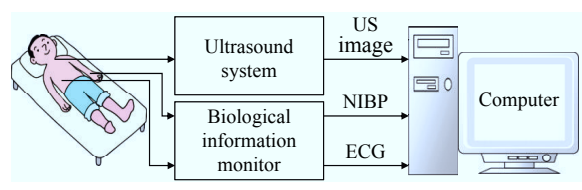

Fig. 2. Experimental equipment for capturing ultrasonographic images and measuring biological signals

Here $\tilde{\eta}$ is viscosity of the arterial wall. From the above, the relationship between the circumferential strain and blood pressure for arteries that are circular in their cross-sectional short axis can be represented by Equation (5) (Fig. 1).

\section{B. Parameter estimation}

Biological signals (electrocardiogram: $E C G(t)$; arterial diameter: $r(t)$; blood pressure: $\left.P_{b}(t)\right)$ were measured simultaneously in order to estimate the parameters by using the linear least-squares method. As the measured signals included noise due to measurement error and the movement of the patient, the frequency characteristics of the vessel diameter waveform and continuous blood pressure were adjusted using a digital filter. Next, using the $\mathrm{R}$ wave of the ECG as a reference, the biological signals of cardiac cycles were retrieved. $t_{0}$ is defined as the timing of the $\mathrm{R}$ wave for each cardiac cycle, so the terms $\ln \left(P_{b}(t) / P_{b}\left(t_{0}\right)\right) \quad \varepsilon(t)$ and $\dot{\varepsilon}(t)$ were calculated using Equation (5). As described above, with electrocardiogram, blood pressure and arterial diameter data, it is possible to estimate stiffness and viscosity on a beat-to-beat basis. The parameters of stiffness $\beta$, viscosity $\eta$ and modified viscosity $\tau=\eta / \beta$ are proposed as log-linearized arterial viscoelastic indices which are of intravascular pressure independence.

\section{EXPERIMENTS}

In order to investigate the validity of the proposed method, we conducted arterial viscoelastic index measurement experiments on several subjects using a one-direction log-linearized arterial viscoelastic model (equivalent to Equation (5)). As previous studies performed by a number of research institutes have concluded that the progression of arteriosclerosis is strongly associated with age [10], we investigated the relationship between the proposed arterial viscoelastic indices and age.

In order to eliminate the influence of gender, 13 male volunteers were chosen for the study. The subjects were divided into two groups - a youth group (5 males, 22.6 $1.5 \mathrm{yrs}$ ) and a middle-aged group ( 8 males, $55.6 \quad 5.3 \mathrm{yrs}$ ). In addition, the middle-aged group were also classified into two groups, that is a pre-existing disease (PED) group and a non-PED group. A subject with PED has one or more of arteriosclerosis, diabetes mellitus, and hyperpiesia. There are no subjects with PED in the youth group. Figure 2 gives an overview of the measurement system. During the experiment, the subjects were supine. To estimate the relevant mechanical properties, blood pressure and the arterial diameter should be measured at the same site. However, as synchronous measurement was difficult due to the shape of the sensor/probe, 


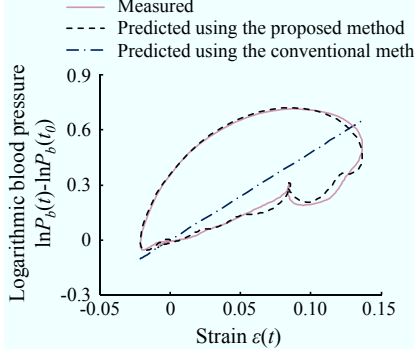

(a)

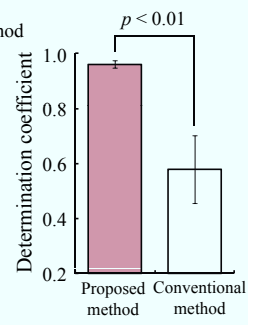

(b)
Fig. 3. Comparison between the proposed method and the conventional method: (a) relationship between measured and predicted blood pressure; (b) determination coefficients

the left common carotid artery was used for ultrasonic measurement, the left radial artery was used for arterial blood pressure measurement, and electrocardiogram signals were measured simultaneously over 10 cardiac cycles. Noninvasive blood pressure (NIBP) and electrocardiogram signals were determined using a biological information monitor (BP608, Omron Colin) with a sampling frequency of $200[\mathrm{~Hz}]$, and an ultrasound system (SONOS5500, Hewlett Packard) was used for ultrasound measurement. Ultrasound image was transferred to an ultrasound acquisition computer with a screen resolution of $640 \times 480$ [pixels] at 30 [fps]. The arterial diameter waveform was measured using the distance between the inner walls as shown by the ultrasound image, and was re-sampled to $200[\mathrm{~Hz}]$ in the first-order hold for synchronization of blood pressure. The proposed indices were estimated using the least-squares method for each cardiac cycle after filtering of the biological signals (a low-pass filter with the cut-off frequency $=10[\mathrm{~Hz}]$ ).

We compared the proposed method and the conventional indicators of arteriosclerosis such as stiffness parameter $\beta$, intima-media thickness (IMT) and brachial ankle pulse wave velocity (baPWV). The stiffness parameter $\beta$ defined by Equation (1) and IMT were calculated from the ultrasound images and blood pressure values used in the evaluation of the proposed method. baPWV was measured using blood pressure pulse wave inspection equipment (form PWV/ABI BP-203, Omron Colin). Data are reported as means \pm S.D. Welch's $t$-test was used to determine the significance of differences between the youth group and the middleaged group for all parameters. Student's $t$-test was used to determine the significance of differences between the evaluation methods. Differences were considered significant when results showing $p<0.05$ were seen.

Informed consent was obtained from all study subjects before the experiments were performed based on the Declaration of Helsinki.

\section{Results}

Figure 3 (a) shows an example of the relationships between circumferential strain and measured blood pressure or predicted blood pressure with the proposed method and predicted blood pressure as determined using the stiffness
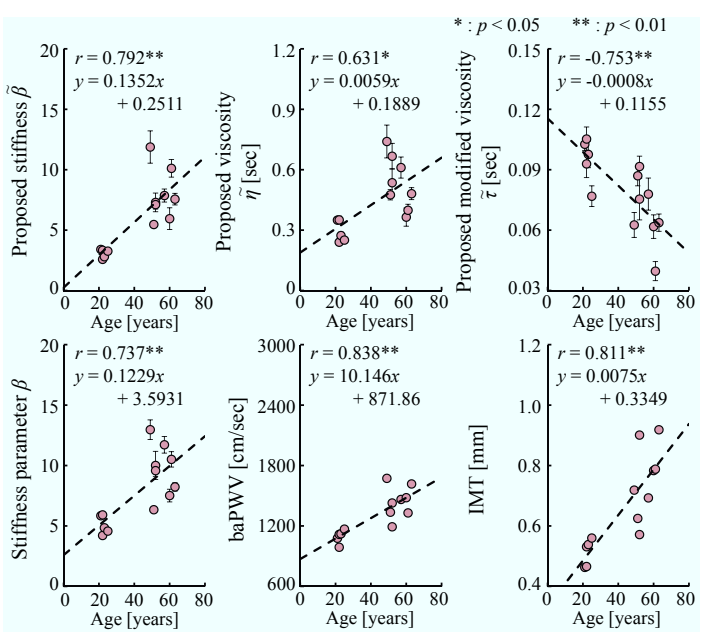

Fig. 4. Relationships between age and estimated indices

parameter $\beta$. The results show that the predicted blood pressure waveform with the proposed method is similar to the Lissajous waveform of the measured signals; the coefficient of determination between them was 0.985. On the other hand, the relationship between strain and predicted blood pressure with the stiffness parameter $\beta$ is linear; the coefficient of determination between them was 0.531 . The coefficients of determination between measured blood pressure and predicted blood pressure with the proposed method and predicted blood pressure with the stiffness parameter $\beta$ for all subjects were $0.960 \quad 0.013$ and $0.578 \quad 0.122$, respectively. These results indicate that the determination coefficient of the proposed method is significantly better than that of the stiffness parameter $\beta$.

Figure 4 shows the relationship between age and the estimated indices of all subjects. The results indicate that stiffness and viscosity increase with age, while modified viscosity decreases. The coefficients of correlation between age and proposed stiffness $\tilde{\beta}$, viscosity $\tilde{\eta}$ and modified viscosity $\tilde{\tau}$ are $0.792,0.631$ and -0.753 , respectively. A positive correlation has also been clinically shown between age and the conventional indicators used to diagnose arteriosclerosis.

Figure 5 shows the relationship between the proposed stiffness $\tilde{\beta}$ and proposed modified viscosity $\tilde{\tau}$. The result indicates that the former is higher in older subjects. In addition, in the middle-aged group with PED, the modified viscosity $\tilde{\tau}$ tends to be lower than that for the healthy middle-aged group with similar values of stiffness $\tilde{\beta}$. Table I shows the results of comparison based on the presence or absence of PED classified according to subjects in the middle-aged group. The results show that the conventional indicators of arteriosclerosis do not differ significantly with or without PED, while the proposed modified viscosity $\tilde{\tau}$ show significant differences.

\section{Discussion}

Figure 3 shows that the Lissajous waveform for predicted blood pressure obtained using the proposed method is similar 


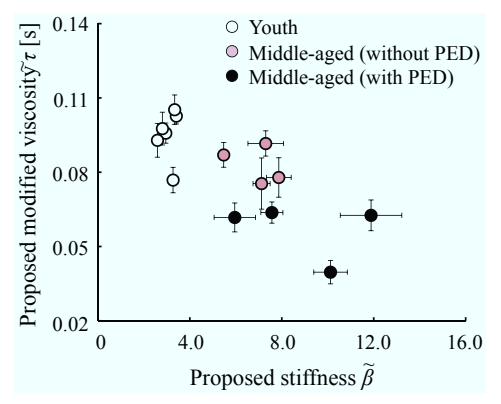

Fig. 5. Two-dimensional plots for all subjects obtained using estimated stiffness and modified viscosity values

to that of the measured waveform, and that the coefficient of determination between measured blood pressure and predicted blood pressure obtained using the proposed method is significantly higher than that for predicted blood pressure obtained using the stiffness parameter $\beta$ for all subjects. The proposed method, which is used to estimate arterial mechanical characteristics including the viscosity of the arterial wall, can therefore be described as more accurate than the previous methods. When arterial mechanical characteristics are estimated using only information on the movement of the arterial wall (such as values of maximum strain and maximum strain velocity), the results depend heavily on intravascular pressure because the nonlinearity between arterial diameter and blood pressure cannot be expressed. It is therefore considered that the proposed dynamic model, which takes into account the nonlinearity and viscoelastic properties of the arterial wall, can be used to provide accurate and detailed clarification for the status of arterial organic diseases.

Furthermore, the modified viscosity $\tilde{\tau}$, which is the ratio of stiffness $\tilde{\beta}$ and viscosity $\tilde{\eta}$, decreases significantly with age. As arterial flexibility is lost with age, it becomes more difficult for the arterial wall to absorb rises in systolic blood pressure, meaning that it cannot expand smoothly. In addition, Table I indicates that the modified viscosity $\tilde{\tau}$ with the proposed method differ significantly between the PED group and the non-PED group. Accordingly, it can be inferred that the proposed method is suitable for detailed estimation of arterial wall viscoelastic properties and enables accurate assessment of changes in the properties of arteries.

\section{CONCLUSION}

In this paper, we proposed a novel log-linearized arterial viscoelastic model for evaluation of the viscoelastic properties of arteries in consideration of intravascular pressure. Using the proposed method, arterial viscoelastic indices were estimated using arterial diameter values measured from ultrasound images and blood pressure.

Then, we conducted arterial viscoelastic index measurement experiments involving the carotid artery with several subjects. The results showed that the estimation accuracy of the proposed method is higher than that achieved using the stiffness parameter $\beta$. The method was also found to support more accurate quantitative evaluation of the mechanical
TABLE I

COMPARISON BETWEEN THE MIDDLE-AGED GROUPS WITH AND WITHOUT PRE-EXISTING DISEASES

\begin{tabular}{|c|c|c|c|}
\hline & $\begin{array}{l}\text { Without PED } \\
\quad(\mathrm{n}=4)\end{array}$ & $\begin{array}{l}\text { With PED } \\
\quad(\mathrm{n}=4)\end{array}$ & $p^{*}$ \\
\hline Mean blood pressure $[\mathrm{mmHg}]$ & $99.5 \pm 15.7$ & $80.9 \pm 34.6$ & N.S. \\
\hline Strain (maximum) & $0.056 \pm 0.019$ & $0.065 \pm 0.029$ & N.S. \\
\hline Strain rate (maximum) $\left[\mathrm{s}^{-1}\right]$ & $0.680 \pm 0.196$ & $0.747 \pm 0.369$ & N.S. \\
\hline Log-linearlized arterial viscoelastic index $\widetilde{\beta}$ & $6.939 \pm 1.030$ & $8.880 \pm 2.636$ & N.S. \\
\hline Log-linearlized arterial viscoelastic index $\tilde{\eta}[\mathrm{s}]$ & $0.572 \pm 0.084$ & $0.496 \pm 0.170$ & N.S. \\
\hline Log-linearlized arterial viscoelastic index $\tilde{\tau}[\mathrm{s}]$ & $0.083 \pm 0.008$ & $0.057 \pm 0.012$ & $<0.05$ \\
\hline Stiffness parameter $\beta$ & $9.413 \pm 2.245$ & $9.807 \pm 2.465$ & N.S. \\
\hline $\mathrm{baPWV}[\mathrm{cm} / \mathrm{s}]$ & $1355 \pm 121$ & $1525 \pm 153$ & N.S. \\
\hline IMT [mm] & $0.698 \pm 0.144$ & $0.802 \pm 0.084$ & N.S. \\
\hline
\end{tabular}

* Significance level for two-tailed Welch's $t$-test of the middle-aged groups with and without PED Values are means \pm S.D. N.S. : not significant $(p>0.05)$

characteristics of arterial walls. This is because it takes into account the fact that viscosity has a strong negative correlation with age and can be discriminated regardless of the presence or absence of pre-existing disease in middleaged subjects.

In this paper, we highlighted the relationship between arteriosclerosis and arterial stiffness/viscosity. In the future, we will attempt to formulate a method of estimation that includes inertia characteristics. In addition, research will be conducted to assess the validity of the proposed method for evaluating arteriosclerosis through comparison with the conventional method of diagnosis and measurement with subjects of various ages.

\section{REFERENCES}

[1] The Ministry of Health Population Movement Statistics 2011, Health, Labour and Welfare Ministry, Tokyo, Japan. [online]. Available http://www.mhlw.go.jp/toukei/saikin/hw/jinkou/geppo/nengai07/kekka3. html (accessed on 20th October 2012)

[2] J. C. Bramwell et al., "Velocity of Transmission of the Pulse Wave," Lancet, vol. 1, pp. 891-892, 1922.

[3] W. W. Nichols, "Clinical Measurement of Arterial Stiffness Obtained from Noninvasive Pressure Waveforms," Am. J. Hypertens., vol. 18, pp. 3S-10S, 2005.

[4] J. I. Davies et al., "Pulse Wave Analysis and Pulse Wave Velocity: a critical review of their strengths and weaknesses," J. Hypertens., vol. 21, no. 3, pp. 463-472, 2003.

[5] I. B. Wilkinson et al., "The influence of heart rate on augmentation index and central arterial pressure in humans," J. Physiol., vol. 525-1, pp. 263-270, 2000.

[6] K. Hayashi et al., "Stiffness and Elastic Behavior of Human Intracranial and Extracranial Arteries," J. Biomech., vol. 13, no. 2, pp. 175-184, 1980.

[7] Y. Tardy et al., "Non-Invasive Estimate of the Mechanical Properties of Peripheral Arteries from Ultrasonic and Photoplethysmographic Measurements," Clin. Phys. Physiol. Meas., vol. 12, no. 1, pp. 39-54, 1991.

[8] J. G. Barra et al., "In vivo angiotensin II receptor blockade and converting enzyme inhibition on canine aortic viscoelasticity," Am. J. Physiol., vol. 272, pp. H859-H868, 1997.

[9] A. Sakane, et al., "Estimating Arterial Wall Impedance Using a Plethysmogram," in 29th Annu. Conf. IEEE Indust. Electro. Soc., USA, 2003, pp. 580-585.

[10] Y. Matsushima et al., "Relationship of carotid intima-media thickness, pulse wave velocity, and ankle brachial index to the severity of coronary artery atherosclerosis," Clin. Cardiol, vol. 27, no. 11, pp. 629-639, 2004. 